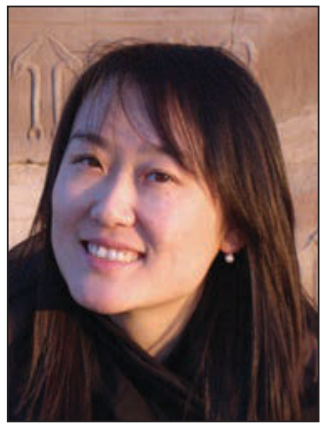

\title{
Laura Na Liu to present The Kavli Foundation Early Career Lectureship in Materials Science
}

aura Na Liu is a professor at the Kirch-
hoff Institute for Physics at Universität Heidelberg, Germany. She received her $\mathrm{PhD}$ degree in physics at Universität Stuttgart in 2009, working on 3D complex plasmonics at optical frequencies. In 2010, she worked as a postdoctoral fellow at the University of California, Berkeley, and in 2011, she joined Rice University as a Texas Instruments Visiting Professor. At the end of 2012, she obtained a Sofja Kovalevskaja Award from the Alexander von Humboldt Foundation and became an independent group leader at the Max Planck Institute for Intelligent Systems. Liu joined Universität Heidelberg in 2015.

Her research interest is multidisciplinary: She works at the interface between nanoplasmonics, biology, and chemistry. Her group focuses on developing sophisticated and smart plasmonic nanosystems for answering structural biology questions as well as catalytic chemistry questions in local environments.

In her presentation at the 2018 Materials Research Society (MRS) Fall Meeting, Liu will discuss a diverse set of DNA-assembled plasmonic nanostructures according to their characteristic optical properties. She will also discuss the evolution from static to dynamic plasmonic systems along with the fast development of this interdisciplinary field.

The Kavli Foundation is dedicated to advancing science for the benefit of humanity, promoting public understanding of scientific research, and supporting scientists and their work.

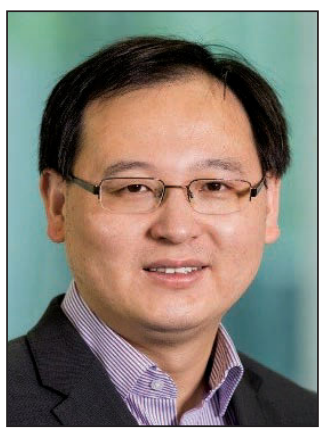

\section{Xinliang Feng to receive the EU-40 Materials Prize}

$\mathrm{T}$ he Materials Research Society (MRS) and the European Materials Research Society (E-MRS) have partnered to highlight the achievements of the EU-40 Materials Prize and the MRS Mid-Career Researcher Award recipients at each respective Society meeting. This E-MRS award is reserved for researchers showing exceptional promise as leaders in materials science, having performed the research for which this prize is awarded while working in Europe.
Xinliang Feng is a full professor and chair of the Molecular Functional Materials Department at Technische Universität Dresden (TU Dresden), Germany. He has published more than 420 research articles and has received the IUPAC Prize for Young Chemists (2009), European Research Council (ERC) Starting Grant Award (2012), Journal of Materials Chemistry Lectureship Award (2013), Chem Comm Emerging Investigator Lectureship
(2014), Fellowship of the Royal Society of Chemistry (2014), Small Young Innovator Award (2017), and the Hamburg Science Award (2017). $\mathrm{He}$ is an Advisory Board member for Advanced Materials, Journal of Materials Chemistry A, ChemNanoMat, Energy Storage Materials, and Small Methods and Chemistry-An Asian Journal. He is the head of the ESF Young Research Group at the Graphene Center at TU Dresden, and working package leader of WP Functional Foams \& Coatings for the European Commission's pilot project "Graphene Flagship."

In his lecture, Feng will present recent efforts on bottom-up synthetic approaches toward novel 1D nanoribbons and organic 2D materials with structural control at the atomic/ molecular level or at the mesoscale. Fundamental electronic, magnetic, and mechanical properties will be discussed.

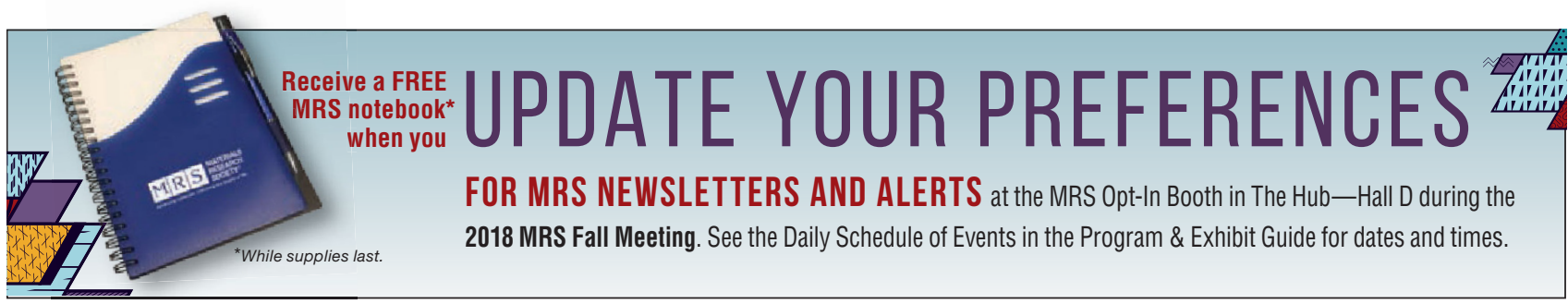

